\title{
Controllable Nanocage Structure Derived from Cyclodextrin-Intercalated Layered Double Hydroxides and Its Inclusion Properties for Dodecylbenzene
}

\author{
Xiao Lei Liu, ${ }^{\dagger}$ Min Wei, ${ }^{* \dagger}{ }^{\dagger}$ Zhong Lin Wang, ${ }^{*}$, David G. Evans, $^{\dagger}$ and Xue Duan ${ }^{\dagger}$ \\ State Key Laboratory of Chemical Resource Engineering, Beijing University of Chemical Technology, \\ Beijing 100029, P.R. China, and Center for Nanoscience and Nanotechnology, School of Materials Science and \\ Engineering, Georgia Institute of Technology, Atlanta, Georgia 30332-0245
}

Received: July 6, 2008; Revised Manuscript Received: September 9, 2008

\begin{abstract}
A novel nanocage structure derived from carboxymethyl- $\beta$-cyclodextrins (CMCDs) intercalated in layered double hydroxides (LDHs), whose gates can be controlled by the process of swelling/drying the CMCD-LDH, has been prepared. Furthermore, the extent of opening of this nanocage structure can be controlled by swelling in different solvents. Dodecylbenzene (DDB) as the guest molecule has been incorporated into the nanocage structure through two different routes: intercalation of CMCD in the LDH followed by inclusion of DDB (intercalation-inclusion method) and inclusion of DDB in CMCD followed by intercalation of the host-guest complex into the LDH (inclusion-intercalation method). For the convenience of using this nanocage as an absorbent and storage vessel for neutral guest, films of the resulting composite materials (CMCD-LDH) were fabricated by the method of solvent evaporation on glass substrates. The structures, chemical compositions, morphologies, and physicochemical properties of the materials were fully studied. Moreover, the effects of the combined confinement of both the LDH layers and the cyclodextrin cavity on the encaged guest were investigated. Compared with the confinement effect produced by cyclodextrin only, this double-confinement imposes stronger restrictions on the mobility of the guest molecule, which leads to a blue shift of the fluorescence spectrum and increases the decay time of the guest. Therefore, this structured nanocage might have potential applications as adsorbents, synergistic agents, and storage vessels for neutral molecules.
\end{abstract}

\section{Introduction}

Over the past 20 years, there has been a surge of interest in nanomaterials, including structures with at least one dimension under $100 \mathrm{~nm} .{ }^{1,2}$ Caging a guest molecule within a nanostructured host lattice produces a confined system with interesting physical and chemical properties. These host systems provide a unique opportunity for exploring the effects of a size-controlled nanoenvironment on the structural and dynamical properties of guest molecules. ${ }^{3,4}$ A direct consequence of confinement is restricted translational and rotational mobilities, or reorientation of the molecule. Additionally, the properties of the materials can be tuned by confining materials on a quantum scale, such as by reducing the dimensions from bulk materials to twodimensional layers,,${ }^{4,5}$ one-dimensional hollows, ${ }^{6}$ and zerodimensional dots. ${ }^{7}$ Recent interests have focused on hollowsphere structures, which can encage large quantities of guest molecules or large-sized guests within the "empty" core domain.

Layered double hydroxides (LDHs, also known as anionic clays) are a class of materials consisting of positively charged brucite-like layers and exchangeable interlayer anions. They have recently received increasing attention, owing to their versatility and usefulness in a wide range of technological applications such as adsorbents, ${ }^{8}$ functional materials, ${ }^{9}$ twodimensional solid-state nanoreactors, ${ }^{10}$ bioactive nanocomposites, ${ }^{11}$ and so on. LDHs can be represented by the general formula $\left[\mathrm{M}^{2+}{ }_{1-x} \mathrm{M}^{3+}{ }_{x}(\mathrm{OH})_{2}\right]^{x+}\left(\mathrm{A}^{n-}{ }_{x / n}\right) \cdot m \mathrm{H}_{2} \mathrm{O}$, where $\mathrm{M}^{2+}$ and $\mathrm{M}^{3+}$ are divalent and trivalent metal cations, respectively, and

* To whom correspondence should be addressed. E-mail: weimin@ mail.buct.edu.cn (M.W.), zhong.wang@mse.gatech.edu (Z.L.W.).

${ }^{\dagger}$ Beijing University of Chemical Technology.

$¥$ Georgia Institute of Technology.
$\mathrm{A}^{n-}$ is an anion. The structure of LDHs is most clearly described by considering the brucite-like structure, $\mathrm{Mg}(\mathrm{OH})_{2}$, which is built up of octahedral units with a $\mathrm{Mg}^{2+}$ ion at the center that is coordinated to hydroxyl groups. In an LDH material, isomorphous replacement of a fraction of the divalent cations by a trivalent cation occurs and generates a positive charge on the layers that necessitates the presence of interlayer, chargebalancing anions. The remaining free space of the interlayer galleries can be occupied by varying numbers of water molecules.

Cyclodextrins (CDs) are one of the most important host molecules for providing nanocavities. They are truncated coneshaped molecules with a hydrophilic outside and a hydrophobic hollow. Because of this structure, CDs form inclusion complexes with various organic molecules. CDs are useful for investigating the effects of spatial restriction because the cavity diameter varies with the number of glucose units constituting the $C D$, e.g., $\alpha-\mathrm{CD}(5 \AA)$ consisting of six units, $\gamma$ - $\mathrm{CD}(6.5 \AA)$ consisting of seven units, and $\gamma$-CD $(8 \AA)$ consisting of eight units. ${ }^{12}$ Modified cyclodextrins have been used as "guest" molecules incorporated into layered hosts such as montmorillonite, ${ }^{13}$ $\alpha$-ziconium phosphate, ${ }^{14}$ and layered double hydroxides. ${ }^{15-17}$ In our previous work, the intercalation of sulfated $\beta$-cyclodextrin and carboxymethyl- $\beta$-cyclodextrin into LDHs and the structural characterization, thermal decomposition behavior, and enantioselective adsorption properties of the resulting materials were reported. ${ }^{18,19}$

In the commonly observed powder form, LDHs have a "house-of-cards" structure involving edge-to-face particle interactions, ${ }^{20}$ which limits their suitability for research and applications. The preparation of LDH films is one of the most effective solutions to this problem. ${ }^{21-24}$ Use of immobilized 


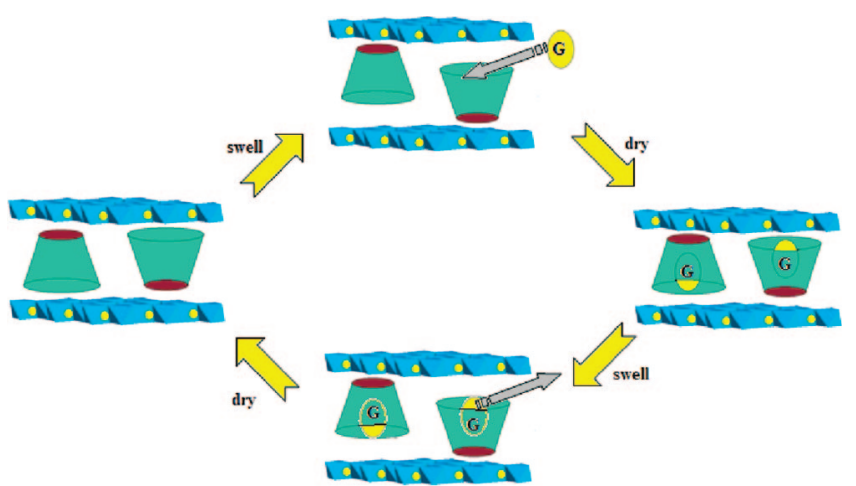

Figure 1. Schematic representation of the controllable opening/closing of the CMCD-LDH nanocage and the cycle of encapsulation into and departure from the nanocage for the guest $(\mathrm{G})$.

LDH nanocrystal films provides several advantages. For example, films can be conveniently used as structured adsorbents, structured catalysts, and other devices. In addition, film allow for the observation of morphological changes in the LDH during reaction.

In this article, we report an easily prepared, controllable nanocage structure formed by carboxymethyl- $\beta$-cyclodextrin (CMCD) nanocavities intercalated in an LDH, as well as its swelling/contraction properties in different solvents. Interestingly, when the $\mathrm{CD}$ nanocage intercalates into the interlayer region of an $\mathrm{LDH}$, both openings of the $\mathrm{CD}$ are blocked by LDH sheets, forming a lockable nanocage. Furthermore, it was found that the opening and closing of the nanocages can be controlled based on the swelling property of the LDH (schematized in Figure 1). When the CMCD-LDH composite is dispersed in solvents such as water or ethanol, the interlayer spacing expands to provide sufficient space for the guest molecule to diffuse from the solution into the nanocavity of CMCD. After the solvent is removed by drying of the sample, the interlayer spacing decreases to its original size, and the guest molecule is thus retained in the nanocavity. The expansion and contraction of the basal spacing lead to the opening and closing of the nanocage and is reversible for repeated swelling/drying cycles. As a result, the cycle of encapsulation into and departure from the nanocage for the guest is also reversible and controllable.

In order to study the properties of the confined nanocage between the sheets of the LDH, we chose dodecylbenzene (DDB) as the model molecule. Two different routes for the synthesis of DDB/CMCD-LDH powder were employed, and a film of the CMCD-LDH nanocage structure was fabricated by the method of solvent evaporation on glass substrates. Moreover, the effects of the combined confinement of both the LDH layers and the CMCD cavity on the encaged guest were investigated. Compared with the confinement effect produced by cyclodextrin only, this double-confinement imposes stronger restrictions on the mobility of the guest molecule, which results in a blue shift of the fluorescence spectrum and increases the decay time of the guest. Therefore, the aim of this work was to provide a procedure for the construction of nanocage structure based on functionalized CD/layered material composites and to present a detailed understanding of how the nanoenvironments of the LDH-intercalated CD cavities influence the photophysics of the included DDB guest molecules. It can be expected that this structured nanocage could be directly applied to adsorb, store, deliver, or interact cooperatively with a wide range of neutral molecules.

\section{Experimental Section}

Reagents. All chemicals including $\mathrm{Zn}\left(\mathrm{NO}_{3}\right)_{2} \cdot 6 \mathrm{H}_{2} \mathrm{O}$, $\mathrm{Al}\left(\mathrm{NO}_{3}\right)_{3} \cdot 9 \mathrm{H}_{2} \mathrm{O}, \mathrm{NaOH}, \mathrm{NaNO}_{3}$, ethanol, $n$-hexane, $N, N$ dimethylformamide, formamide, $\beta$-cyclodextrin $(\beta$-CD), and DDB were of analytical grade and were used without further purification. $\beta$-CD and DDB were purchased from Aldrich; the others were obtained from Beijing Chemical Plant Limited.

Syntheses. Synthesis of CMCD. CMCD was synthesized according to the procedure described in the literature, ${ }^{25}$ with some modifications. In brief, $\mathrm{CD}(32.4 \mathrm{~g})$ and $\mathrm{NaOH}(35.2 \mathrm{~g})$ were dissolved in $200 \mathrm{~mL}$ of water, and monochloroacetic acid $(37.8 \mathrm{~g})$ was added with gentle stirring. The mixture was heated at $80{ }^{\circ} \mathrm{C}$ for $3 \mathrm{~h}$. The solution was then cooled in an ice bath, and the $\mathrm{pH}$ was adjusted to 6.0 with concentrated hydrochloric acid. Methanol was gradually added to the solution with stirring to obtain CMCD precipitate. The average number of carboxylate groups (4.1) per CMCD molecule was determined using ${ }^{1} \mathrm{H}$ NMR spectroscopy. ${ }^{26}$

Synthesis of DDB/CMCD-LDH Powder. DDB/CMCDLDH powder was prepared by two different methods.

In the intercalation-inclusion method, the precursor $\mathrm{NO}_{3}-\mathrm{LDH}$ was prepared by a coprecipitation method similar to that reported previously. ${ }^{27} \mathrm{CMCD}-\mathrm{LDH}$ was obtained by the method of ion exchange. A solution of CMCD $(2.5 \mathrm{~g})$ in deionized water $(50 \mathrm{~mL})$ was added to a suspension of $\mathrm{NO}_{3}-\mathrm{LDH}(10.0 \mathrm{~g})$ in water $(100 \mathrm{~mL})$, and the $\mathrm{pH}$ of the solution was kept at 6.0 by adding $0.1 \mathrm{~mol} / \mathrm{L} \mathrm{NaOH}$ solution or $0.1 \mathrm{~mol} / \mathrm{L} \mathrm{HNO}_{3}$ solution during the reaction. The mixture was heated at $60{ }^{\circ} \mathrm{C}$ under a nitrogen atmosphere for $48 \mathrm{~h}$. The product was washed extensively with deionized water, centrifuged, and stored to be used later in an inclusion reaction. DDB was included within CMCD-LDH by reaction with an ethanol solution. In a typical inclusion experiment, $0.10 \mathrm{~mL}$ of DDB was allowed to equilibrate with suspension of CMCD-LDH $(9.5 \mathrm{~g})$ in ethanol $(100 \mathrm{~mL})$ with stirring at $70{ }^{\circ} \mathrm{C}$ for $48 \mathrm{~h}$. The solid was washed thoroughly with ethanol, centrifuged, and dried at $70{ }^{\circ} \mathrm{C}$ for $20 \mathrm{~h}$. This product is denoted P1.

In the inclusion-intercalation method, a mixture of two solutions, one consisting of CMCD $(2.0 \mathrm{~g})$ in water $(20 \mathrm{~mL})$ and the other consisting of DDB $(0.08 \mathrm{~mL})$ in ethanol $(50 \mathrm{~mL})$, was refluxed at $60^{\circ} \mathrm{C}$ for $48 \mathrm{~h}$ under vigorous magnetic stirring to obtain the DDB/CMCD inclusion complex. A sample of $8.0 \mathrm{~g}$ of $\mathrm{NO}_{3}-\mathrm{LDH}$ was added to this solution, and then the mixture was stirred at $70{ }^{\circ} \mathrm{C}$ for $48 \mathrm{~h}$. The solid was washed extensively with ethanol and water, centrifuged, and dried at $70{ }^{\circ} \mathrm{C}$ for $20 \mathrm{~h}$. This sample is denoted P2.

Synthesis of DDB/CMCD-LDH Films. In the intercalationinclusion method, a CMCD-LDH film was prepared by applying an aqueous $\mathrm{CMCD}-\mathrm{LDH}$ suspension (1.0 wt \%) to a glass substrate and then drying it at $70{ }^{\circ} \mathrm{C}$ for $20 \mathrm{~h}$. All glass substrates were pretreated in an aqueous $\mathrm{H}_{2} \mathrm{O}_{2} / \mathrm{NH}_{4} \mathrm{OH}(3: 7 \mathrm{v} / \mathrm{v})$ solution for 30 minand then rinsed thoroughly with deionized water. The CMCD-LDH film was dipped into $30 \mathrm{~mL}$ of an ethanol solution of DDB $(0.26 \% \mathrm{v} / \mathrm{v})$ in a microwave oven. After an initial ramp program from ambient temperature to $110{ }^{\circ} \mathrm{C}$, the solution was maintained at this temperature for $120 \mathrm{~min}$ by appropriate choice of microwave oven power. After the sample had been cooled to room temperature, the film on the glass substrate was washed thoroughly with ethanol and dried at 70 ${ }^{\circ} \mathrm{C}$ for $20 \mathrm{~h}$. This sample is denoted F1.

In the solvent evaporation method, an aqueous suspension of $\mathrm{DDB} / \mathrm{CMCD}-\mathrm{LDH}(\mathrm{P} 2)$, after dilution to $2.8 \mathrm{wt} \%$ at $\mathrm{pH} 6$, was dripped onto a glass substrate and dried in air at $70{ }^{\circ} \mathrm{C}$ for $20 \mathrm{~h}$. This sample is denoted F2. 


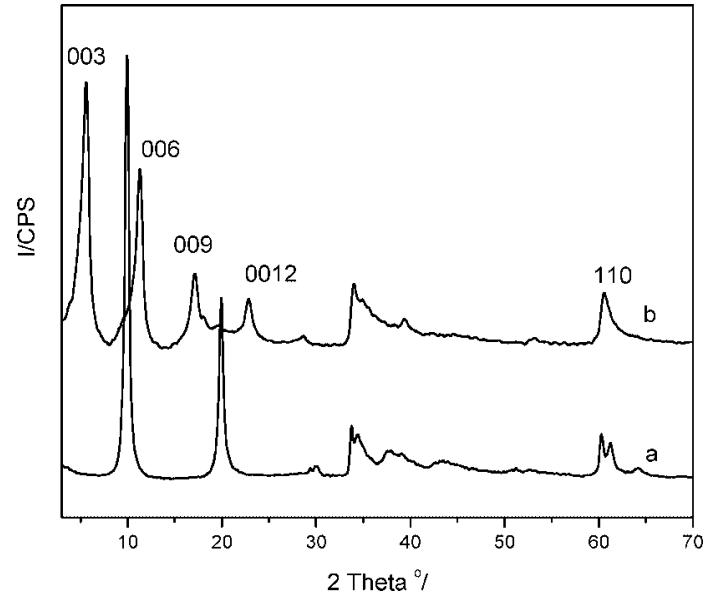

Figure 2. Powder XRD patterns of (a) $\mathrm{NO}_{3}-\mathrm{LDH}$, (b) CMCD-LDH.

Swelling of CMCD-LDH in Solvents. Dried CMCD-LDH $(1.0 \mathrm{~g})$ was separately dispersed in $100 \mathrm{~mL}$ of various solvents, such as water, methanol, ethanol, $n$-propanol, and $n$-butanol, by a 15 -min ultrasonic treatment and then refluxed at $45^{\circ} \mathrm{C}$ for $48 \mathrm{~h}$. The white precipitates obtained were collected by centrifugation.

Characterization. Powder X-ray diffraction (XRD) patterns of the samples were recorded using a Shimadzu XRD-6000 diffractometer with $\mathrm{Cu} \mathrm{K} \alpha$ radiation $(\lambda=1.5406 \AA)$. Simultaneous thermogravimetry - mass spectrometry (TG-MS) analyses were performed with a Pyris Diamond thermogravimetrydifferential thermal analysis (TG-DTA) instrument coupled to a ThermoStar QM220 mass spectrometer by a quartz capillary transfer line at $180^{\circ} \mathrm{C}$. The heating rate was $10^{\circ} \mathrm{C} \mathrm{min}^{-1}$, with a $\mathrm{N}_{2}$ flow of $100 \mathrm{~mL} \mathrm{~min}{ }^{-1}$. The scanning speed was $1 \mathrm{amu}$ $\mathrm{s}^{-1}$, with a filtering time of $0.03 \mathrm{~s}$. The TG apparatus operated at atmospheric pressure, and the mass spectrometer operated at a working pressure of $3 \times 10^{-6} \mathrm{mPa}$ and an electron energy of $70 \mathrm{eV}$. Metal analysis was performed by inductively coupled plasma (ICP) emission spectroscopy on a Shimadzu ICPS-7500 instrument using solutions prepared by dissolving the samples in dilute $\mathrm{HCl}$. Carbon, hydrogen, and nitrogen analyses were carried out using an Elementarvario elemental analysis instrument. Scanning electron microscopy (SEM) images were obtained using a Hitachi S-4700 scanning electron microscope. Fluorescence spectra were recorded on a Shimadzu RF-530PC instrument. Fluorescence decay measurements were carried out at room temperature on an Edinburgh FLS920 time-resolved and steady-state fluorescence spectrometer, equipped with a continuous-wave 450-W xenon arc lamp as the excitation source for steady-state measurements and a nanosecond flash lamp for lifetime measurements using the time-correlated single-photoncounting technique. The flash lamp operated at a pulse frequency of $40 \mathrm{kHz}$ with a typical pulse half-width of $1 \mathrm{~ns}$. All decays were measured with a range of $100 \mathrm{~ns}$, and counts at the maximum were about 3000 in all decays. The adequacy of the nonlinear least-squares fit was judged by the reduced $\chi^{2}$ value $(<1.2$ in all cases).

\section{Results and Discussion}

CMCD-LDH as a Controllable Nanocage. The XRD patterns of $\mathrm{NO}_{3}-\mathrm{LDH}$ and $\mathrm{CMCD}-\mathrm{LDH}$ are displayed in Figure 2, and the lattice parameters are listed in Table S1 of the Supporting Information. All of the patterns indicate formation of a well-crystallized hydrotalcite-like LDH phase. In each

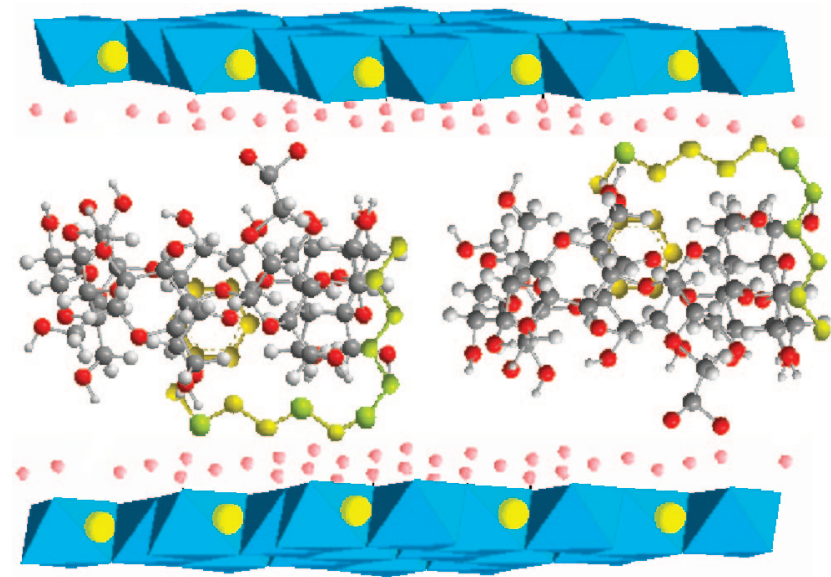

Figure 3. Schematic representation of the possible arrangement of $\mathrm{DDB} / \mathrm{CMCD}-\mathrm{LDH}$.

case, the reflections can be indexed to a hexagonal lattice with $R 3 m$ rhombohedral symmetry, commonly used for the description of LDH structures. ${ }^{28,29}$ The intercalation of CMCD in the lamellar host structure was clearly evidenced by the increase in the $d_{003}$ basal spacing from $0.884 \mathrm{~nm}$ for the precursor $\mathrm{NO}_{3}-\mathrm{LDH}$ to $1.610 \mathrm{~nm}$ for CMCD-LDH. The corresponding gallery heights were calculated to be 0.404 and $1.130 \mathrm{~nm}$, respectively, by subtracting the thickness of the inorganic layer $(0.48 \mathrm{~nm}) .^{30}$ Taking into account the dimensions of the carboxymethyl-modified $\beta$-cyclodextrin molecule (CD molecules should be regarded as truncated cones with an outer diameter of $1.53 \mathrm{~nm}^{12}$ and a height of $1.06 \mathrm{~nm}^{15}$ ), the CMCD molecule must adopt a monolayer arrangement with its cavity axis perpendicular to the LDH host layer (shown in Figure 3).

The XRD patterns of the CMCD-LDH samples swollen in water, methanol, ethanol, $n$-propanol, and $n$-butanol are summarized in Figure S1 of the Supporting Information. It was found that the interlayer distance $\left(d_{003}\right)$ of CMCD-LDH increases significantly after the material is swollen. As reported by other researchers, ${ }^{31,32}$ the expansion of the interlayer distance increases the system energy, whereas the associated interlayer reorganization and hydrogen bonding can decrease it. Therefore, the formation of a hydrogen-bonding network among the host layers, interlayer anions, and solvent molecules plays an important role in the swelling process. In the case of dry CMCD-LDH, the hydrogen-bonding network exists mainly between the metal-hydroxide layers and the CMCD anions. After the material is swollen in various solvents, the solvent molecules diffuse into the interlayer region and participate in the formation of the hydrogen-bonding network. It was found in this work that the expansion of interlayer spacing decreases with increasing size of the hydrophobic part of the solvent (from $2.338 \mathrm{~nm}$ in water to $1.632 \mathrm{~nm}$ in $n$-butanol, as shown in Figure S1 of the Supporting Information). This trend is expected, because the hydrophobic tails interact unfavorably both with the hydrophilic $\mathrm{LDH}$ layers and with the exterior of CMCD. Therefore, it is disadvantageous for a solvent with weak polarity to be involved in the formation of the interlayer hydrogen-bonding network. Figure S2 (Supporting Information) shows that the $d_{003}$ values of the redried CMCD-LDH samples are quite close to that of fresh CMCD-LDH $(1.610 \mathrm{~nm})$, demonstrating the recovery property of swollen LDH. As a result, the opening/closing of the interlayer CMCD nanocage can be controlled by repeating swelling/drying cycles. 


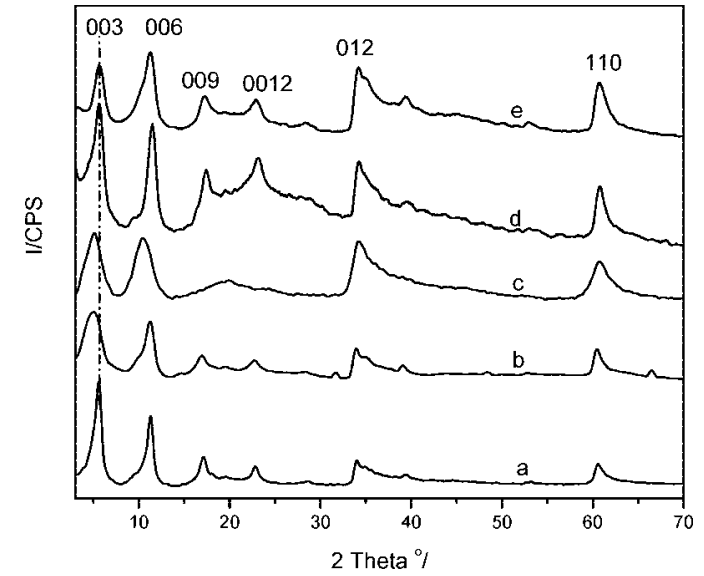

Figure 4. Powder XRD patterns of (a) CMCD-LDH, (b) P1, (c) P2, (d) reclaimed CMCD $-\mathrm{LDH}$ from $\mathrm{P} 1$, and (e) reclaimed CMCD-LDH from P2.

Inclusion Ability of the CMCD-LDH Nanocage. $X$-ray Diffraction. The XRD patterns of CMCD-LDH, DDB/ CMCD-LDH (both P1 and P2), and reclaimed CMCD-LDH (from both P1 and P2) are shown in Figure 4, and the lattice parameters are listed in Table S1 of the Supporting Information. It was found that, upon inclusion of DDB (Figure 4b,c), the value of $d_{003}$ of CMCD-LDH increased from 1.610 to 1.804 and $1.778 \mathrm{~nm}$ for $\mathrm{P} 1$ and $\mathrm{P} 2$, respectively, corresponding to increases of 0.194 and $0.168 \mathrm{~nm}$. According to a comparison between the increase in gallery height and the molecular length of DDB (1.93 nm, calculated using Chemwindow 6.0) as well as the inclusion property of $\mathrm{CDs}$, it can be concluded that the phenyl moiety of a DDB molecule is included in the cyclodextrin cavity, whereas the alkyl chain remains outside the cavity. A schematic representation of the possible arrangement for DDB/CMCD-LDH is shown in Figure 3. To gain insight into the process of releasing DDB from this nanocage, we subsequently reclaimed the CMCD-LDH by stirring $\mathrm{P} 1$ or $\mathrm{P} 2$ in water/ethanol (2:5) solution for $48 \mathrm{~h}$, and the XRD patterns are displayed in Figure 4d,e. An obvious shift of the (003) reflection to higher $2 \theta$ value can be observed for the two reclaimed CMCD-LDH samples, with $d_{003}$ values reduced to 1.581 and $1.560 \mathrm{~nm}$, respectively, which are close to that of fresh CMCD-LDH $(1.610 \mathrm{~nm})$. The UV-vis spectrum of the filtrate after the release experiment is shown in Figure S3b of the Supporting Information. The spectrum shows band at about 256 $\mathrm{nm}$, which is in accordance with the characteristic of the pristine DDB (Figure S3a, Supporting Information). This indicates that the DDB molecules depart from the opened nanocages in the water/ethanol solution during the releasing process.

Figure S4 (Supporting Information) shows the XRD patterns of the CMCD-LDH and DDB/CMCD-LDH films (F1 and F2) on glass substrates, which are significantly different from those of the corresponding powder samples (Figure 4). The sequence of strong $(00 l)$ peaks up to $l=15$ at low angle and the absence of any nonbasal reflections $(h, k \neq 0)$ at high angle for the films are characteristic of extremely well $c$-oriented assemblies of LDH platelets. The basal spacing of the
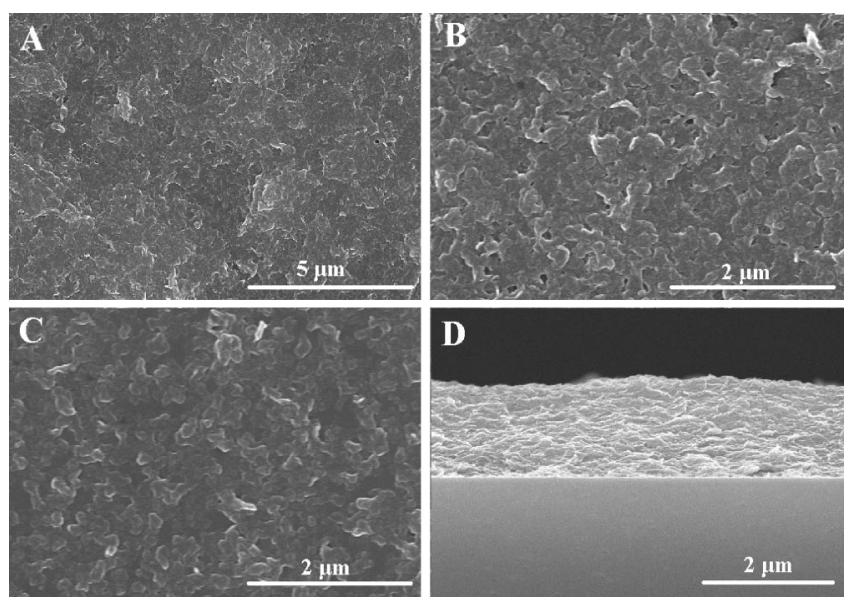

Figure 5. SEM images of (A) CMCD-LDH film, (B) F1, (C) F2, (D) edge view of $\mathrm{F} 2$.

CMCD-LDH film sample is $1.659 \mathrm{~nm}$, close to the value of the corresponding powder sample. However, the basal spacing increases to $1.710 \mathrm{~nm}(\mathrm{~F} 1)$ and $1.732 \mathrm{~nm}(\mathrm{~F} 2)$ after reaction with DDB, indicating that the alkyl benzene has been successfully included into the cavity of CMCD.

Simultaneous thermogravimetry (TG) and differential thermal analysis (DTA) combined with analysis by mass spectrometry (MS) also confirms the inclusion of DDB by CMCD-LDH. Figure S5 and S6 (Supporting Information) display the TG-DTA profiles and TG-MS traces, respectively, for DDB/CMCDLDH. Table S2 (Supporting Information) lists the proposed mass numbers and ion current intensities of the observed species. A detailed discussion can be obtained in the Supporting Information.

Elemental Analysis. The chemical compositions of the synthesized powder products $\mathrm{NO}_{3}-\mathrm{LDH}, \mathrm{CMCD}-\mathrm{LDH}$, and $\mathrm{DDB} / \mathrm{CMCD}-\mathrm{LDH}(\mathrm{P} 1$ and $\mathrm{P} 2)$ are listed in Table 1. As shown in Table 1, the experimentally determined $\mathrm{Zn} / \mathrm{Al}$ molar ratio of CMCD-LDH is approximately equal to that of the $\mathrm{NO}_{3}-\mathrm{LDH}$ precursor, and it decreased slightly after the inclusion of DDB into the CMCD-functionalized LDH. The extent of inclusion of DDB in the CMCD-LDH was found to correspond to a ratio of 0.400 and 0.525 molecules of DDB per anchored CMCD cavity in $\mathrm{P} 1$ and $\mathrm{P} 2$, respectively. The higher molar ratio of DDB to CMCD in P2 than in P1 indicates that the inclusionintercalation route is more effective than the intercalationinclusion route in achieving the inclusion of DDB in CMCD - LDH.

SEM. SEM images of the three film samples, CMCD-LDH, F1, and F2, are shown in Figure 5. In all cases, the films exhibit a mostly flat surface in the view (Figure $5 \mathrm{~A}-\mathrm{C}$ ). The individual LDH platelets, with diameters of ca. $200 \mathrm{~nm}$, are densely packed in the two-dimensional plane of the film. A high degree of $c$-axis orientation of the LDH platelets is observed, consistent with the XRD data (Figure S4, Supporting Information). The edgeview image of F2 (Figure 5D) shows that the thickness of the film is about $2 \mu \mathrm{m}$ with anisotropic structural features. The CMCD-LDH and F1 films also exhibit similar uniformly densely packed $c$-oriented arrangements of platelets (edge views

TABLE 1: Chemical Compositions of $\mathrm{NO}_{3}-\mathrm{LDH}, \mathrm{CMCD}-\mathrm{LDH}$, and DDB/CMCD-LDH (P1 and P2)

\begin{tabular}{lcl}
\hline \multicolumn{1}{c}{ sample } & $\mathrm{Zn} / \mathrm{Al}$ & \multicolumn{1}{c}{ chemical composition } \\
\hline $\mathrm{NO}_{3}-\mathrm{LDH}$ & 2.03 & $\mathrm{Zn}_{0.67} \mathrm{Al}_{0.33}(\mathrm{OH})_{2.00}\left(\mathrm{NO}_{3}\right)_{0.33} \cdot 0.8 \mathrm{H}_{2} \mathrm{O}$ \\
$\mathrm{CMCD}-\mathrm{LDH}$ & 1.94 & $\mathrm{Zn}_{0.66} \mathrm{Al}_{0.34}(\mathrm{OH})_{2.00}(\mathrm{CMCD})_{0.043}\left(\mathrm{NO}_{3}\right)_{0.18} \cdot 2.6 \mathrm{H}_{2} \mathrm{O}$ \\
$\mathrm{P} 1$ & 1.86 & $\mathrm{Zn}_{0.65} \mathrm{Al}_{0.35}(\mathrm{OH})_{2.00}(\mathrm{CMCD})_{0.045}(\mathrm{DDB})_{0.018}\left(\mathrm{NO}_{3}\right)_{0.18} \cdot 1.9 \mathrm{H}_{2} \mathrm{O}$ \\
$\mathrm{P} 2$ & 1.86 & $\mathrm{Zn}_{0.65} \mathrm{Al}_{0.35}(\mathrm{OH})_{2.00}(\mathrm{CMCD})_{0.040}(\mathrm{DDB})_{0.021}\left(\mathrm{NO}_{3}\right)_{0.20} \cdot 1.7 \mathrm{H}_{2} \mathrm{O}$
\end{tabular}



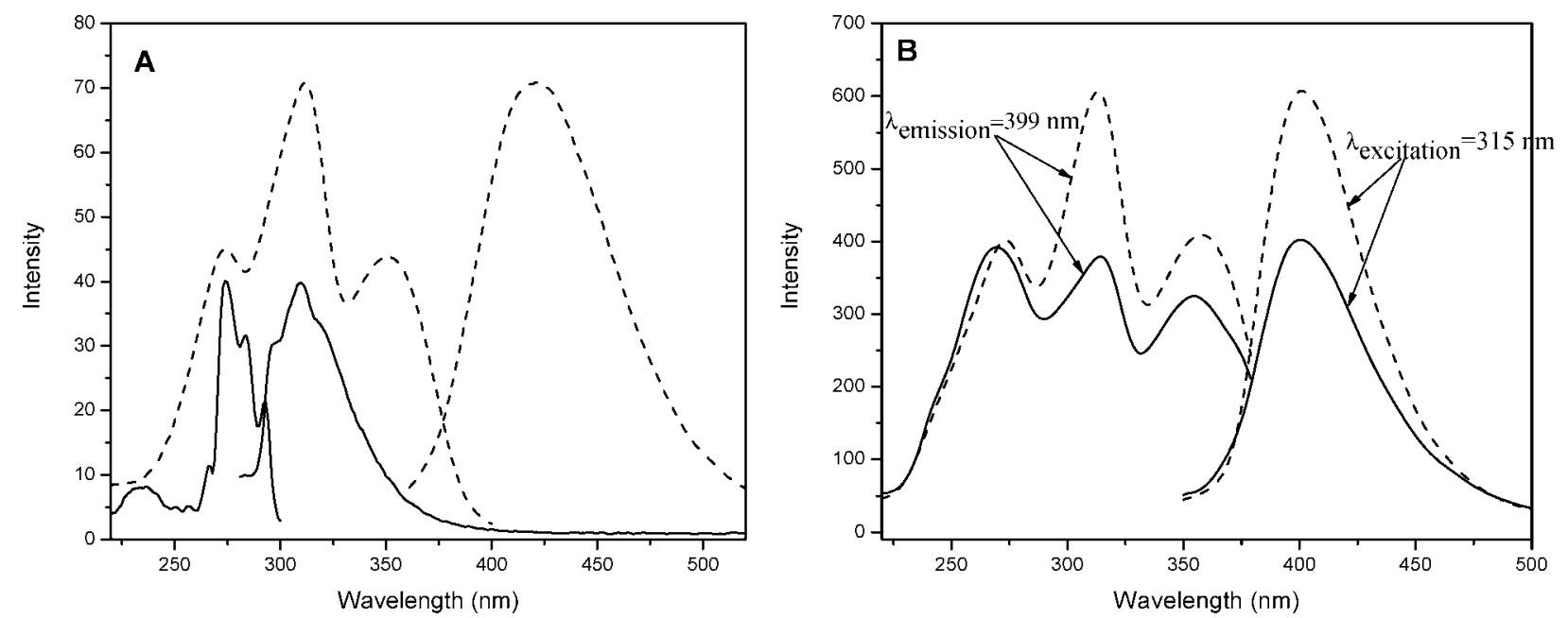

Figure 6. Fluorescence excitation and emission spectra of (A) DDB in ethanol solution (solid line), DDB/CMCD in water/ethanol (2:5) solution (dashed line); (B) P1 (solid line), P2 (dashed line).

not shown). LDH particles are platelets with a tendency to lie on their largest surface to share surface anions between platelets. Therefore, the face-to-face type of aggregation of platelets is predominant in these LDH films, similar to other reports of the formation of LDH films on substrates.

Space-Confinement Effect of the Nanocage on DDB. Study of Fluorescence Properties. Any molecule containing delocalized $\pi$ electrons such as DDB has a reasonable chance of being fluorescent. The fluorescence excitation and emission spectra of DDB in ethanol (solid line) and of the DDB/CMCD inclusion complex in water/ethanol solution (dashed line) are shown in Figure 6A. The positions of the bands for both the excitation and emission spectra of DDB are similar to those of benzene..$^{33}$ The excitation peak of DDB at around $235 \mathrm{~nm}$ (Figure 6A, solid line) is associated with the $\mathrm{S}_{0} \rightarrow \mathrm{S}_{2}$ transition. The spectral features at 274, 284, and $293 \mathrm{~nm}$ in the excitation spectra are due to excitation from the ground state $\left(S_{0}\right)$ to different vibrational energy levels of the first electronic excited state $\left(S_{1}\right)$. The corresponding emission $\left(S_{1} \rightarrow S_{0}\right)$ in the fluorescence spectrum appears at $310 \mathrm{~nm}$. In comparison with DDB itself, the intensities of the peaks in the spectrum of DDB/CMCD in water/ethanol (2:5) are enhanced, with emission and excitation peak maxima moving to longer wavelengths (Figure 6A, dashed line). After inclusion in CMCD, the main excitation bands $\mathrm{S}_{0}$ $\rightarrow \mathrm{S}_{2}(\sim 312 \mathrm{~nm})$ and $\mathrm{S}_{0} \rightarrow \mathrm{S}_{1}(\sim 350 \mathrm{~nm})$ shift by $\sim 77$ and $\sim 66 \mathrm{~nm}$, respectively, to longer wavelength compared with those of the pristine DDB in ethanol, whereas the absorption band $\mathrm{S}_{0} \rightarrow \mathrm{S}_{3}$ transition appears at about $274 \mathrm{~nm}$. Moreover, a red shift of $\sim 114 \mathrm{~nm}$ in the fluorescence emission band (424 $\mathrm{nm}$ ) of DDB is observed after inclusion in CMCD, which is considered to be clear evidence for the formation of an inclusion complex. The spectral shifts indicate that the excited states of the caged DDB are in a more stable structure upon encapsulation. Interactions between a guest molecule and its surroundings can broaden the vibrational bands of the spectra, and the degree of broadening is indicative of the strength of the moleculesurroundings interaction. ${ }^{34}$ In contrast to DDB itself, the vibrational bands in the fluorescence spectra of DDB/CMCD cannot be resolved, indicating that the interactions of the guest with its surroundings increase after inclusion.

In general, interpretation of the band shift in the fluorescence spectrum with different surroundings is complicated, because the observed changes can be the results of several quite different interactions that might either augment or minimize each other. ${ }^{35-39}$ To gain a clearer insight into the significance of the various effects of the surroundings, the fluorescence and absorption spectra of DDB in solvents of different dielectric constants and of DDB after inclusion in unsubstituted CD and CMCD in water/ethanol solution were recorded. Figure S7 (Supporting Information) shows the emission spectra of DDB in $n$-hexane, ethanol, $N, N$-dimethylformamide (DMF), and formamide and of DDB included in CD and CMCD in water/ ethanol solution. The emission bands of DDB in the first four solvents are observed at 283, 309, 342, and $405 \mathrm{~nm}$, respectively. The red shift of the emission wavelength is well-correlated with the corresponding dielectric constant $(n$-hexane $<$ ethanol $<$ $\mathrm{DMF}<$ formamide). In the cases of inclusion by $\mathrm{CD}$ and CMCD, the emission bands of DDB move to 413 and $424 \mathrm{~nm}$, respectively. The results demonstrate that binding of DDB to $\mathrm{CD}$ and $\mathrm{CMCD}$ changes the local environment in a way that is similar to increasing the dielectric constant. There have been many debates over the dielectric constant inside cyclodextrin nanocavities. Several authors ${ }^{40,41}$ have estimated the dielectric constant of cyclodextrin on the basis of absorption or fluorescence spectral data such as peak shifts or peak intensity ratios of guest molecules used as probes. The reported data range widely ( $\epsilon=5.0-74)$, depending on the host - guest combination. This variation in the effective dielectric constant in CD cavities can be explained by the size difference between the guest molecule and the cavity diameter. DDB is a small aromatic molecule, containing only one phenyl group. When CD incorporates DDB, other small molecules such as water or ethanol can also be included in the cavity. As a result, the effective dielectric constant is expected to be large, consistent with the observed fluorescence spectra. The emission spectrum of DDB/ CMCD (at $424 \mathrm{~nm}$ ) shows a red shift of $11 \mathrm{~nm}$ compared to that of $\mathrm{DDB} / \mathrm{CD}$ (at $413 \mathrm{~nm}$ ), indicating that the introduction of the carboxylate substituent leads to an increase in the dielectric constant of the CD cavity.

Figure 6B displays the fluorescence excitation and emission spectra of DDB included in CMCD that has been intercalated in the restricted gallery region of $\mathrm{LDH}$ (P1, solid line; $\mathrm{P} 2$, dashed line). It can be seen that the band positions in the fluorescence excitation and emission spectra of $\mathrm{P} 1$ and $\mathrm{P} 2$ are rather close. The excitation bands at around 276, 315, and $358 \mathrm{~nm}$ are associated with $\mathrm{S}_{0} \rightarrow \mathrm{S}_{3}, \mathrm{~S}_{0} \rightarrow \mathrm{S}_{2}$, and $\mathrm{S}_{0} \rightarrow \mathrm{S}_{1}$ transitions, respectively. Obvious band broadening can be observed, and no vibrational bands can be resolved. The emission spectra of 


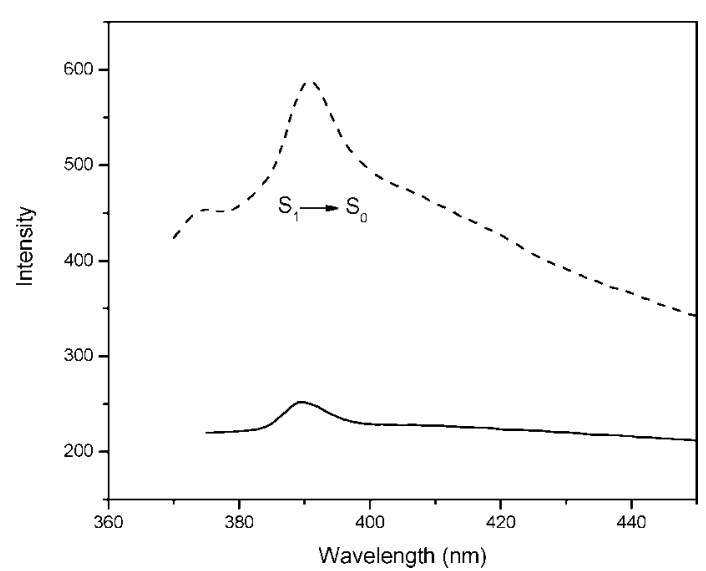

Figure 7. Fluorescence emission spectra of DDB/CMCD-LDH films on glass substrates (F1, solid line; F2, dashed line).

both P1 and P2 display the characteristic emission peak at 399 $\mathrm{nm}$, representing a significant blue shift compared with that of DDB/CMCD in solution (424 nm). Most probably, the shift to shorter wavelength is reminiscent of the effects of enhanced restriction. As the nanocavities of CMCD are enclosed by LDH sheets, the effects of restriction on the included DDB are induced not only by the cyclodextrin cavities, but also by the LDH sheets in the opening of CMCD. This double-confinement imposes stronger restrictions on the rotation and translation of the guest molecule, as well as the increased interactions between guest and host. Therefore, an obvious blue shift in the emission spectrum for DDB/CMCD-LDH can be detected.

To study the influence of the DDB content on the fluorescence properties, the fluorescence emission spectra of $\mathrm{P} 2$ were recorded with different molar ratios of DDB to CMCD ranging from 0.05 to 0.51 (see Figure S8, Supporting Information). It was found that no significant band shift could be observed (all appear at ca. $399 \mathrm{~nm}$ ) with increasing DDB/CMCD ratio, whereas the fluorescence intensity increased continuously. The explanation for this behavior is straightforward. The cyclodextrin cavities are anchored to the LDH host sheets and thus lack translational mobility. These anchored CMCD cavities prevent aggregation of DDB molecules, so that the intermolecular interaction in this system is rather weak. Therefore, the influence of DDB content on the shift of the fluorescence emission band is negligible.

The emission spectra of DDB/CMCD-LDH films on glass substrates (F1 and F2) are shown in Figure 7. The emission band $\left(\mathrm{S}_{1} \rightarrow \mathrm{S}_{0}\right)$ of included DDB appears at $391 \mathrm{~nm}$. Compared to those of the powder samples, the emission bands of the films shift to slightly shorter wavelengths, which might be due to the enhanced effect of the constrained geometry after the formation of the film. This result is in accordance with the XRD study, in which the basal spacing of the film samples is less than that of the powder samples, indicating that a straighter nanocage formed in the film.

Fluorescence Decay Measurements. Time-resolved fluorescence decay measurements can provide more direct information about the environment around DDB. The fluorescence decay data were fitted to the equation

$$
F(t)=\sum_{i=1}^{n} A_{i} \exp \left(-t / \tau_{i}\right)
$$

where $n=1$, 2, etc.

The fluorescence decay profiles were recorded for excitation at $272,312,315$, and $340 \mathrm{~nm}$ with emission monitored at 310 , 424,399 and $391 \mathrm{~nm}$ for DDB, DDB/CMCD, DDB/

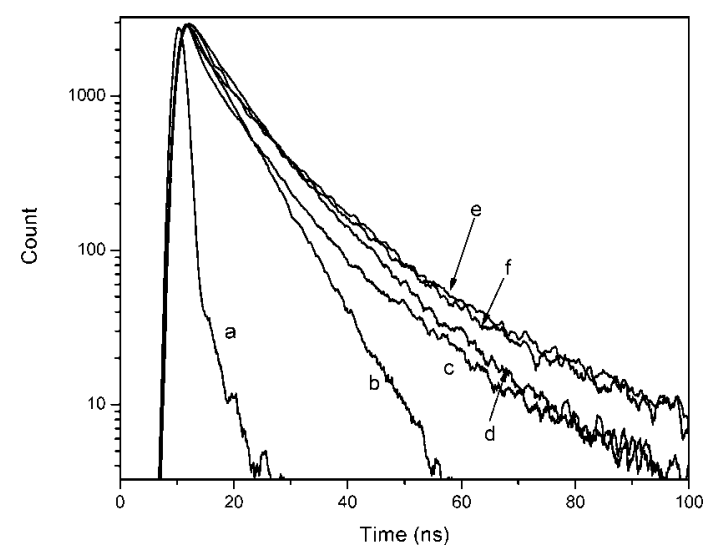

Figure 8. Time-resolved fluorescence decay of (a) fit, (b) DDB in ethanol, (c) DDB/CMCD in water/ethanol, (d) P2, (e) P1, (f) F2.

TABLE 2: Emission Lifetimes and Normalized Pre-exponential Factors from the Multiexponential Fit of the Fluorescence Decays of DDB, DDB/CMCD, and DDB/ CMCD-LDH

\begin{tabular}{lccccccc}
\hline \multicolumn{1}{c}{ sample } & $\begin{array}{c}\lambda_{\mathrm{ex}} \\
(\mathrm{nm})\end{array}$ & $\begin{array}{c}\lambda_{\mathrm{em}} \\
(\mathrm{nm})\end{array}$ & $\begin{array}{c}\tau_{1} \\
(\mathrm{~ns})\end{array}$ & \multicolumn{1}{c}{$A_{1}$} & $\begin{array}{c}\tau_{2} \\
(\mathrm{~ns})\end{array}$ & $A_{2}$ & $\chi^{2}$ \\
\hline DDB & 272 & 310 & 6.51 & 1 & - & - & 1.071 \\
DDB/CMCD & 312 & 424 & 2.79 & 0.948 & 9.95 & 0.052 & 1.157 \\
P1 & 315 & 399 & 7.30 & 0.959 & 18.44 & 0.041 & 1.029 \\
P2 & 315 & 399 & 7.20 & 0.931 & 15.22 & 0.069 & 1.132 \\
F2 & 340 & 391 & 7.64 & 0.914 & 17.49 & 0.086 & 1.031
\end{tabular}

CMCD-LDH powder sample (both P1 and P2), and F2, respectively. It should be explained that, because the fluorescence intensity of F1 was too weak, the fluorescence lifetime could not be measured. The results are shown in Figure 8.

The fluorescence lifetimes $(\tau)$ and pre-exponential factors $(A)$ for DDB in ethanol, DDB/CMCD in water/ethanol, P1, P2, and F2 are summarized in Table 2. It was found that the decay curve for DDB in ethanol gives a good fit to a single-exponential function. In contrast, the decay curves for DDB/CMCD in water/ ethanol, for DDB/CMCD-LDH (both P1 and P2), and for F2 could be fitted not to a single-exponential function but to a linear combination of two exponential functions. The two-component decay indicates that the DDB molecules are located in two different environments and that the interconversion of the two species is much slower than the fluorescence decay, which occurs on the nanosecond time scale.

The reason for the double-exponential fluorescence lifetime of $\mathrm{DDB}$ in $\mathrm{DDB} / \mathrm{CMCD}-\mathrm{LDH}$ could be the presence of free DDB molecules in the LDH gallery space in a dynamic equilibrium with included DDB given that the free DDB would presumably have different decay kinetics from the complexed DDB. However, this can be ruled out by the following experiment: A sample of $\mathrm{NO}_{3}-\mathrm{LDH}$ was added to a solution of DDB in ethanol and stirred at $70{ }^{\circ} \mathrm{C}$ for $48 \mathrm{~h}$ (the same reaction conditions as for inclusion of DDB in CMCD-LDH). No fluorescence signal could be obtained for the resulting sample, indicating that the hydrophobic DDB was unable to diffuse into the hydrophilic LDH gallery. Another explanation for the double-exponential decay could be a mixture of $1: 1$ and 1:2 DDB/CMCD complexes between the layers of LDH, with each of them showing different decay kinetics. This idea can also be eliminated, because CMCD can adopt only a perpendicular monolayer-like arrangement (see the section X-ray Diffraction) and, thus, formation of a DDB/CMCD complex with 1:2 stoichiometry is not feasible. 
An alternative explanation is that both DDB environments involve insertion into the CD host, as it was previously shown that insertion of substituted phenyl compounds into CDs might give a mixture of two or more alternative forms. ${ }^{42,43}$ Based on our experiments and previous studies, the observed kinetics for $\mathrm{DDB}$ in DDB/CMCD-LDH can be explained by a dynamic equilibrium between DDB molecules that are tightly bound to CMCD (with a relatively long fluorescence decay time) and those that are located near the exit of the hydrophobic cavity (with a relatively short fluorescence decay time).

It can be observed from Table 2 that the difference in emission lifetimes of P1, P2, and F2 is small, whereas their values $\{\mathrm{P} 1$ [7.30 ns $(95.9 \%), 18.44 \mathrm{~ns}(4.1 \%)], \mathrm{P} 2$ [7.20 ns (93.1\%), $15.22 \mathrm{~ns}(6.9 \%)], \mathrm{F} 2$ [7.64 ns $(91.4 \%), 17.49 \mathrm{~ns}$ $(8.6 \%)]$ are significantly larger than those of DDB/CMCD in solution [2.79 ns (94.8\%), $9.95 \mathrm{~ns}(5.2 \%)]$. This result further confirms the effect of the locked nanocages on the stabilization of the excited state of the caged DDB. In the case of DDB included in CMCD that is locked by LDH sheets, the doubleconfinement effect intensively decreases the radiationless rate constant due to twisting motion and thus increases the fluorescence lifetime of the encaged DDB. Therefore, the fluorescence decay lifetimes of DDB/CMCD-LDH are longer than those of $\mathrm{DDB} / \mathrm{CMCD}$ in water/ethanol solution. A comparison between the powder and film samples reveals that the fluorescence lifetimes of F2 are longer than those of P2 and a larger proportion of included DDB in F2 adopts the tightly caged mode compared to that in $\mathrm{P} 2$. This result indicates that the confinement effect of the constrained geometry is enhanced after the formation of the film with a high degree of $c$-axis orientation of the LDH platelets, which is consistent with the blue shift of the bands in the fluorescence spectrum of F2.

\section{Conclusions}

A novel nanocage structure derived from CMCD intercalated in LDH was studied in this work. The opening/closing of the nanocage can be controlled by the process of swelling/drying the CMCD-LDH in various solvents. In both powder and film samples, CMCD molecules adopt a monolayer arrangement, with the axis of the cavity perpendicular to the LDH sheets, and DDB molecules are driven into these hydrophobic cavities by partitioning from a polar solvent. The XRD results show that the basal spacing of the resulting DDB/CMCD-LDH material is larger than that of $\mathrm{CMCD}-\mathrm{LDH}$, and the pyrolysis fragments of DDB appear above $450{ }^{\circ} \mathrm{C}$ in the MS trace of $\mathrm{DDB} / \mathrm{CMCD}-\mathrm{LDH}$. These results all confirm the inclusion of $\mathrm{DDB}$ in the $\mathrm{CMCD}$ nanocavities in the interlayer galleries. Compared with the DDB/CMCD complex in solution, the DDB included in $\mathrm{CMCD}-\mathrm{LDH}$ is embedded in the locked $\mathrm{CMCD}$ nanocage, and the restriction effect is induced by both the cyclodextrin cavities and the LDH sheets in the opening of CMCD. This double-confinement imposes stronger restrictions on the mobility of the guest molecule, which causes a blue shift in the fluorescence spectrum and increases the decay time. The decay times can be fitted by a double-exponential function, indicating that the DDB molecules are accommodated in two different inclusion environments, i.e., one loosely bound and the other tightly bound. Therefore, this work provides a controllable nanocage based on a functionalized CD/layered material composite that could be directly applied to adsorb, store, deliver, or interact cooperatively with a wide range of neutral molecules.

Acknowledgment. This work was supported by the National Natural Science Foundation of China, the Program for New
Century Excellent Talents in University (Grant NCET-05-121), the 973 Program (Grant 2009CB939802), and the Program for Changjiang Scholars and Innovative Research Team in University (Grant IRT0406).

Supporting Information Available: XRD patterns of dry CMCD-LDH and CMCD-LDH swollen in water, methanol, ethanol, $n$-propanol and $n$-butanol (Figure S1); XRD patterns of dry CMCD-LDH and redried CMCD-LDH after being swollen in water, methanol, ethanol, $n$-propanol and $n$-butanol (Figure S2); UV-vis spectra of the filtrate after the release of DDB from DDB/CMCD-LDH (Figure S3); XRD patterns of the CMCD-LDH film, F1, and F2 on glass substrates (Figure S4); TG-DTA profiles of DDB/CMCD-LDH heated under nitrogen (Figure S5); TG-MS traces for DDB/CMCD-LDH heated under nitrogen (Figure S6); fluorescence emission spectra of DDB in $n$-hexane, ethanol, DMF, formamide, CD, and CMCD (Figure S7); fluorescence emission spectra of P2 with different concentrations of included DDB (Figure S8); indexing of XRD patterns for $\mathrm{NO}_{3}-\mathrm{LDH}, \mathrm{CMCD}-\mathrm{LDH}, \mathrm{DDB} /$ CMCD-LDH (P1 and P2), and the reclaimed samples after the release test (Table $\mathrm{S} 1$ ); results for $\mathrm{MS}$ analysis of $\mathrm{DDB} /$ CMCD-LDH (Table S2). This material is available free of charge via the Internet at http://pubs.acs.org.

\section{References and Notes}

(1) Kale, B. B.; Baeg, J. O.; Apte, S. K.; Sonawane, R. S.; Naik, S. D.; Patil, K. R. J. Mater. Chem. 2007, 17, 4297.

(2) Elder, A.; Yang, H.; Gwiazda, R.; Teng, X.; Thurston, S.; He, H.; Oberdörster, G. Adv. Mater. 2007, 19, 3124.

(3) Gournis, D.; Jankovic, L.; Maccallini, E.; Benne, D.; Rudolf, P.; Colomer, J. F.; Sooambar, C.; Georgakilas, V.; Prato, M.; Fanti, M.; Zerbetto, F.; Sarova, G. H.; Guldi, D. M. J. Am. Chem. Soc. 2006, 128, 6154.

(4) Desigaux, L.; Belkacem, M. B.; Richard, P.; Cellier, J.; Leone, P.; Cario, L.; Leroux, F.; Taviot-Gueho, C.; Pitard, B. Nano Lett. 2006, 6, 199.

(5) Xu, Z. P.; Braterman, P. S. J. Phys. Chem. C 2007, 111, 4021.

(6) Murugavel, R.; Sathiyendiran, M.; Pothiraja, R.; Walawalkar, M. G.; Mallah, T.; Riviere, E. Inorg. Chem. 2004, 43, 945.

(7) Li, J.; Wang, L. W. Chem. Mater. 2004, 16, 4012. 8623 .

(8) Lv, L.; He, J.; Wei, M.; Duan, X. Ind. Eng. Chem. Res. 2006, 45,

(9) Fogg, A. M.; Williams, G. R.; Chester, R.; O'Hare, D. J. Mater. Chem. 2004, 14, 2369.

(10) Gerardin, C.; Kostadinova, D.; Sanson, N.; Coq, B.; Tichit, D. Chem. Mater. 2005, 17, 6473.

(11) Darder, M.; López-Blanco, M.; Aranda, P.; Leroux, F.; Ruiz-Hitzky, E. Chem. Mater. 2005, 17, 1969.

(12) Szejtli, J. Chem. Rev. 1998, 98, 1743.

(13) Kijima, T.; Tanaka, J.; Matsui, Y. Nature 1984, 310, 45.

(14) Kijima, T.; Matsui, Y. Nature 1986, 322, 533.

(15) Zhao, H. T.; Vance, G. F. J. Chem. Soc., Dalton Trans. 1997, 11, 1961

(16) Choy, J. H.; Jung, E. Y.; Son, Y. H.; Park, M. J. Phys. Chem. Solids 2004, 65, 509.

(17) Mohanambe, L.; Vasudevan, S. J. Phys. Chem. B 2005, 109, 22523.

(18) Wang, J.; Wei, M.; Rao, G. Y.; Evans, D. G.; Duan, X. J. Solid State Chem. 2004, 177, 366.

(19) Liu, X. L.; Wei, M.; Li, F.; Duan, X. AIChE J. 2007, 53, 1591. (20) Gursky, J. A.; Blough, S. D.; Luna, C.; Gomez, C.; Luevano, A. N.; Gardner, E. A. J. Am. Chem. Soc. 2006, 128, 8376.

(21) Gardner, E.; Huntoon, K. M.; Pinnavaia, T. J. Adv. Mater. 2001, 13,1263

(22) Lee, J. H.; Rhee, S. W.; Jung, D. Y. Chem. Mater. 2006, 18, 4740. (23) Li, L.; Ma, R. Z.; Ebina, Y.; Fukuda, K.; Takada, K.; Sasaki, T. J. Am. Chem. Soc. 2007, 129, 8000.

(24) Chen, H. Y.; Zhang, F. Z.; Fu, S. S.; Duan, X. Adv. Mater. 2006, 18,3089

(25) Park, S. Y.; Park, J. K.; Park, J. H.; McNeff, C. V.; Carr, P. W. Microchem. J. 2001, 70, 179.

(26) Ravoo, B. J.; Darcy, R.; Mazzaglia, A.; Nolan, D.; Gaffney, K. Chem. Commun. 2001, 827.

(27) Meyn, M.; Beneke, K.; Lagaly, G. Inorg. Chem. 1993, 32, 1209.

(28) Kobayashi, Y.; Ke, X. L.; Hata, H.; Schiffer, P.; Mallouk, T. E.

Chem. Mater. 2008, 20, 2374. 
(29) Pisson, J.; Taviot-Gueho, C.; Israeli, Y.; Leroux, F.; Munsch, P.; Itie, J. P.; Briois, V.; Morel-Desrosiers, N.; Besse, J. P. J. Phys. Chem. B 2003, 107, 9243.

(30) Kwon, T.; Tsigdinos, G. A.; Pinnavaia, T. J. J. Am. Chem. Soc. 1988, $110,3653$.

(31) Kumar, P. P.; Kalinichev, A. G.; Kirkpatrick, R. J. J. Phys. Chem. C 2007, 111, 13517 .

(32) Hou, X. Q.; Kirkpatrick, R. J. Chem. Mater. 2000, 12, 1890.

(33) Muller, D. J.; Knight, A. E. W. J. Phys. Chem. 1984, 88, 3392.

(34) Lumb, M. D. Luminescence Spectroscopy. Acadamic Press: London, 1978.

(35) Grabner, G.; Rechthaler, K.; Mayer, B.; Köhler, G. J. Phys. Chem. A 2000, 104, 1365.

(36) El-Kemary, M.; Organero, J. A.; Santos, L.; Douhal, A. J. Phys. Chem. B 2006, 110, 14128 .
(37) Pimentel, G. C. J. Am. Chem. Soc. 1957, 79, 3323.

(38) Wenska, G.; Koput, J.; Pedzinski, T.; Marciniak, B.; Karolczak, J.; Golankiewicz, B. J. Phys. Chem. A 2006, 110, 11025.

(39) Mukhopadhyay, M.; Banerjee, D.; Mukherjee, S. J. Phys. Chem. A 2006, 110, 12743 .

(40) Madrid, J. M.; Mendicuti, F.; Mattice, W. L. J. Phys. Chem. B 1998, 102, 2037.

(41) Funasaki, N.; Ishikawa, S.; Neya, S. J. Pharm. Sci. 2001, 90, 740.

(42) Duveneck, G. L.; Sitzmann, E. V.; Eisenthal, K. B.; Turro, N. J. J. Phys. Chem. 1989, 93, 7166.

(43) Rüdiger, V.; Eliseev, A.; Simova, S.; Schneider, H. J.; Blandamer, M. J.; Cullis, P. M.; Meyer, A. J. J. Chem. Soc., Perkin Trans. 2 1996, 2119 .

JP805951Z 